\title{
Bridging Cognitive Models and Recommender Systems
}

\author{
Cecilio Angulo ${ }^{1}$. Ing. Zoe Falomir ${ }^{2} \cdot$ Davide Anguita $^{3} \cdot$ Núria Agell $^{4} \cdot$ Erik Cambria $^{5}$
}

Published online: 9 March 2020

(C) Springer Science+Business Media, LLC, part of Springer Nature 2020

Intelligent systems must be designed to interact with human beings. Hence, ideal systems for interacting with people would be those capable of interpreting their environment cognitively, that is, similarly to how people do it.

This special issue proposed a space for researchers to discuss the issues and advantages of bridging different fields for the study of cognitive approaches for recommendation [1-4], and for the development of recommender systems shaping cognitive architectures $[5,6]$.

In recent conferences and meetings [7, 8], we realized that the link between these fields has not received sufficient attention. In our opinion, recommendations should also be designed and evaluated considering cognitive factors. The level of cognitive reasoning in a system or the number of creative solutions that a system can provide can be considered as a measure to evaluate the intelligence of that system $[9,10]$. This issue is closely related with key trends for emerging technologies such as cognitive expert advisors, machine learning or smart robots.

This bridge should be travelled in both sides. From one side, cognition represents an ideal model to link different disciplines, such as robotics, interaction studies, decision support systems and information retrieval. Cognitive architectures are providing recommendations to agents (a human or a robot) in order to interact with the environment (perceived environment or collected data).

Furthermore, by getting assessment from collaborative human expertise, expert recommender systems can automate procedures and tasks in a cognitive way, for physical agents,

Cecilio Angulo

cecilio.angulo@upc.edu

1 Universitat Politècnica de Catalunya - UPC BarcelonaTECH, Barcelona, Spain

2 Universität Bremen, Bremen, Germany

3 Università degli Studi di Genova, Genoa, Italy

4 ESADE - Ramon Llull University, Barcelona, Spain

5 Nanyang Technological University, Singapore, Singapore like robots or soft agents to collect information from databases or Internet.

Hence, topics covered by this special issue are related with cognitive approaches to data decision-making that are context-aware and employ reasoning in several application domains [11]. In this special issue, four papers have been selected out of all the received submissions. These proposals are briefly summarized as follows.

The paper "Perceptions or Actions? Grounding How Agents Interact Within a Software Architecture for Cognitive Robotics" by R. Marfil, A. Romero-Garces, J. P. Bandera, L. J. Manso, L. V. Calderita, P. Bustos, A. Bandera, J. Garcia-Polo, F. Fernandez and D. Voilmy proposes to join the perceptual and acting perspectives in cognitive robotics via a unique representation where the responses of all software modules in the architecture are generalized using the same set of tokens. In this form, the cognitive architecture establishes a close coupling between deliberative and reactive control flows, and robots can react properly upon encountering unexpected changes in their environment that are not part of their planned course of actions.

In the paper "Doctor Recommendation Based on an Intuitionistic Normal Cloud Model Considering Patient Preferences" by Y. Yang, J. Hu, Y. Liu and X. Chen, Chinese medical websites helping patients search for satisfactory doctors via the Internet regardless of time and location are considered. Authors propose a systematic decision support model to improve standard recommendations using intuitionistic fuzzy sets (IFSs) with the Bonferroni mean (BM) to address interdependencies. The obtained system accommodates patient preferences using multiple intuitionistic normal clouds (INCs). A case study is provided using production data from haodf.com, the largest Chinese medical website.

The paper "Creating, Interpreting and Rating Harmonic Colour Palettes Using a Cognitively Inspired Model" by L.Museros, I. Sanz, Z. Falomir and L. Gonzalez-Abril introduces a cognitively inspired qualitative theory, QCharm, which defines five operators for colour combination based on the qualitative colour descriptor (QCD) and applies these 
operators to recommend palettes of harmonic colours. Furthermore, a regression model is implemented to learn users' preferences based on the COLOURlovers dataset. The resulting model is used as an additional criterion for recommendation.

The paper "A Cognitively Inspired Clustering Approach for Critique-Based Recommenders" by D. Contreras and M. Salamó describes how to add a clustering process to a critiquebased recommender, thereby adapting the recommendation process and how a cognitive user preference model can be defined based on the preferences (i.e. defined by critiques) received by the user. Several proposals are developed based on clustering. Obtained results indicate that introducing clustering into the critique-based recommender is an appealing option since it enhances overall efficiency, especially with a large data set.

The guest editors of this special issue hope that the presented contributions will trigger further advances of bridging cognitive models and recommender systems. Finally, we also would like to deeply thank to all the authors for their contributions and the reviewers for their outstanding cooperation, especially for their effort in evaluating the papers considered, and for giving highly constructive feedback to the authors. We also thank A. Hussain, present Editor-in-Chief of the Cognitive Computation journal, for providing us with the opportunity to edit this special issue.

Funding Information Z. Falomir thanks the Cognitive Qualitative Descriptions and Applications (CogQDA) project funded by the University of Bremen and the YERUN Research Mobility Award (Young European Research UNiversities, second edition, 2018/2019).

\section{References}

1. Olteteanu A-M, Falomir Z, Freksa C. Artificial cognitive systems that can answer human creativity tests: an approach and two case studies. IEEE Transactions on Cognitive and Developmental Systems, in press. 2017. https://doi.org/10.1109/TCDS.2016. 2629622
2. Puigbo J-Y, Pumarola A, Angulo C, Tellez R. Using a cognitive architecture for general-purpose service robot control. Connection Science, Taylor \& Francis. 2015. https://doi.org/10.1080/ 09540091.2014.968093.

3. Costa V, Dellunde P, Falomir Z. The logical style painting classifier based on Horn clauses and explanations (1-SHE). Logic J IGPL, special issue on Logic-based applications in AI. On-line first. https://doi.org/10.1093/jigpal/jzz029.

4. Falomir Z, Costa V, Gonzalez-Abril L. Obtaining discriminative colour names according to the context: using a fuzzy colour model and probabilistic reference grounding, international journal of uncertainty, fuzziness and knowledge-based systems. Special issue on Modelling Decisions in AI. 2019;27(Suppl. 1):107-42. https://doi. org/10.1142/S0218488519400063.

5. Ruiz FJ, Raya C, Samà A, Agell N. A transformational creativity tool to support chocolate designers. Pattern Recogn Lett. 2015;67(Part 1):75-80. https://doi.org/10.1016/j.patrec.2015.05. 012.

6. Chiclana F, Agell N, Wu J, Herrera-Viedma E. Fuzzy decisionmaking and consensus: new trends and real-life applications. Appl Soft Comput. 2015;35(October 2015):789-91. https://doi. org/10.1016/j.asoc.2015.07.043.

7. Falomir Z. Towards cognitive image interpretation - qualitative descriptors, domain knowledge and narrative generation. In: Gibert K, Botti V, Reig-Bolaño R, editors. Artificial intelligence research and development, Frontiers in artificial intelligence and applications, vol. 256: IOS Press; 2013. p. 77-86, ISBN 978-161499-319-3, ISSN 0922-6389. https://doi.org/10.3233/978-161499-320-9-77.

8. Kothakota SK, Angulo C. Completing ambiguous plans in cognitive robotics with delayed information. 19th International Conference of the Catalan Association of Artificial Intelligence (CCIA 2016), pp. 19-28, Barcelona, Spain. IOS Press. 2016. https://doi.org/10.3233/978-1-61499-696-5-19

9. Olteteanu A-M, Falomir Z. Object replacement and object composition in a creative cognitive system. A computational counterpart of the alternative use test. Cogn Syst Res. 2016;39:15-32. https:// doi.org/10.1016/j.cogsys.2015.12.011.

10. Falomir Z, Olteteanu A-M. Special issue on problem-solving, creativity and spatial reasoning. Cogn Syst Res. 2019;58:31-4. https:// doi.org/10.1016/j.cogsys.2019.05.001.Editorial.

11. Li Y, Wang S, Pan Q, Peng H, Yang T, Cambria E. Learning binary codes with neural collaborative filtering for efficient recommendation systems. Knowl-Based Syst. 2019;172:64-75.

Publisher's Note Springer Nature remains neutral with regard to jurisdictional claims in published maps and institutional affiliations. 